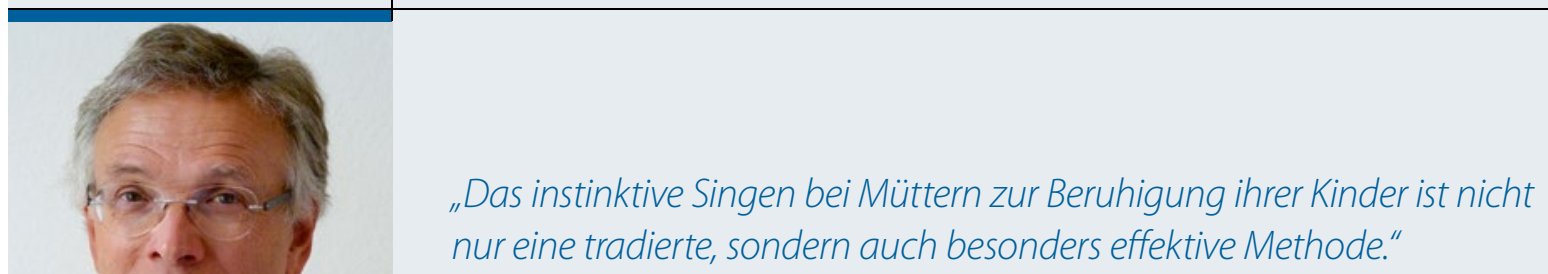

Dr. Ulrich Mutschler, Chefredakteur

Babys beruhigen

\title{
Reden ist Silber, Singen ist Gold
}

— Die emotionale Selbstkontrolle ist bei kleinen Kindern noch wenig entwickelt. Singen kann dabei helfen, die Babys bei der Entspannung zu unterstützen. In einer kanadischen Studie wurden 30 Kinder im Alter von sechs und neun Monaten untersucht [Mariève Corbeil M et al. Infancy 2015; Sep 22]. Die Kinder wurden von ihren Müttern getrennt und es wurde geschaut, welchen Einfluss entweder Musik oder Gespräche auf diesen Stress ausübten. Um den Einfluss von bekannten Melodien auszuschließen, wurden den Kindern türkische Lieder vorgespielt. Hierbei blieben die Kinder für circa 9 Minuten entspannt, während sie beim Sprechen nur für 4-5 Minuten ruhig blieben. Es machte dabei auch keinen Unterschied, ob es sich um Babysprache oder normale Umgangssprache handelte. Das instinktive Singen bei Müttern zur Beruhigung ihrer Kinder ist also nicht nur eine tradierte, sondern auch besonders effektive Methode.

\section{Geheimnis gelüftet}

\section{Warum knacken eigentlich Fingergelenke?}

— An der kanadischen Universität von Edmonton gelang es mithilfe eines Hochgeschwindigkeits-MRT, das bislang ungelöste Rätsel knackender Gelenke zu ergründen [Kawchuk GN et al. PLoS One 2015;10: e0119470]. Einer der Wissenschaftler selber konnte nämlich allen seinen zehn Fingergrundgelenken nach Belieben Geräusche entlocken. Im speziellen MRT mit über drei
Bildern pro Sekunde zeigten die Aufnahmen, dass sich mit zunehmendem Ziehen ein Hohlraum zwischen den Gelenkflächen bildet und dass dabei das Geräusch entsteht. Bis dato hatte man angenommen, dass es erst später, nämlich beim Wiederzusammentreffen der Gelenkflächen, knackt. Unklar bleibt aber dennoch, warum das Knacken trotz der kleinen Gelenke doch recht laut ist.

\section{Teratogene Wirkung \\ Riskante Medikamente für Mädchen}

— US-amerikanische Ärzte aus Kansas City analysierten die Daten von knapp 1.700 Mädchen und Frauen im Alter von 14 bis 25 Jahren, die zwischen 2008 und 2012 insgesamt 4.500 Rezepte für teratogene Medikamente erhalten hatten [Stancil SL et al. Pediatrics 2016; 137: 1-8]. Knapp 70\% der jungen Frauen hatten dabei keinerlei Informationen über ein mögliches Risiko im Falle einer Schwangerschaft mitgeteilt bekommen. Die riskantesten Medikamente waren etwa Topiramat (gegen Epilepsie und auch Migräne), Methotrexat bei malignen und anderen schwerwiegenden Erkrankungen oder Isotretinoin bei schwerer Akne. Am häufigsten klärten dabei noch die Dermatologen mit $47 \%$ auf, während Neurologen mit 16\% wesentlich schlechter abgeschnitten - möglicherweise konnten sie sich Teenagerschwangerschaften gar nicht vorstellen.

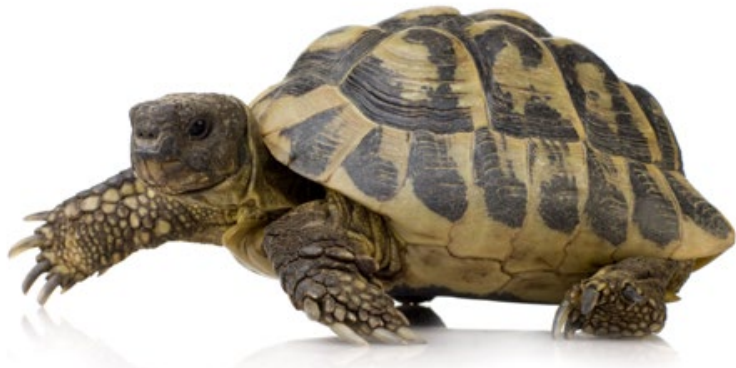

Vorsicht im Umgang mit Reptilien Salmonellen-Gefahr durch Schildkröten

— Schildkröten-assoziierte Salmonellosen sind eine zunehmende Problematik bei Kindern. In den USA konnten in den Jahren 2011-2013 acht Ausbrüche mit 473 Fällen in 41 Bundesstaaten registriert werden. Kinder unter 5 Jahren waren besonders oft betroffen. Salmonellen sind bei Schildkröten physiologische, meist apathogene Bewohner ihres Darms und werden beim Reinigen der Terrarien oder beim Spielen mit den Tieren übertragen. Die Autoren einer US-amerikanischen Studie empfehlen die Versorgung von kleinen Schildkröten nur durch ältere Kinder und Erwachsene und vor allem auch eine penible Handhygiene [Walters MS et al. Pediatrics 2016;137:1-9]. Gefährdete Personengruppen (z.B. Kinder unter 2 Jahren, immungeschwächte Personen, Schwangere) sollten besonders vorsichtig im Umgang mit den Tieren sein. Salmonellen können auch bei anderen Reptilien vorkommen. Durch intensiven Kontakt mit den Tieren oder Freilauf in der Wohnung, und nicht nur durch ihre Exkremente, kann man sich also infizieren. Die weitere Ansteckung erfolgt dann über den oralen Weg, das heißt während des Spielens mit Reptilien sollte man selbst auch nicht essen oder trinken und anschließend die Hände mit Seife waschen. Eltern sollten ihre Kinder öfters auf die erforderlichen Hygienemaßnahmen ansprechen. Sollte das Tier auch selbst eine Salmonelleninfektion haben, kann sich dies anders als beim Menschen in Form von Stomatitis, Dermatitis oder Dystrophie äußern. 